\title{
RELATIONSHIP BETWEEN GENES AT THE PEA AND SINGLE COMB LOCUS AND ECONOMIC TRAITS IN BROILER CHICKEN
}

\author{
W. Hartmann. - Bundesforschungsanstalt für Kleintievzucht, 31 Celle, Dörnbergstr. 25/27. BRD
}

Six hatches of pedigreed broilers, comprising a total of 7593 offsprings from 223 Cornish type sires, II 2 homozygous and III heterozygous for pea comb, were used to study the effect of comb type on final weight, breast blister frequency and carcass yield. Contrary to results reported by Collins et al. (I96I, Poultry Sci,. 40, 824-826) there was no evidence of an association between comb type and broiler growth.

Single comb broilers showed a lower indice of breast blisters than their sibs having pea combs. This difference was highly significant in males and less so in female broilers. It is suggested that this might be another manifestation of the pea comb allel, possibly as a result of structural changes in the skin. At equal weight, female broilers had a significantly lower frequency of breast blisters than males, indicating a direct influence of sex on breast blister formation apart from the immediate effect of body weight.

Broilers with pea comb had a slightly better carcass yield than single comb broilers.

\section{Aspects génétiques de l'hỵpertrophie musculaire des Bovins (earaetère culard)}

\author{
VARIATION DE FRÉQUENCE DU CARACTÈRE CULARD \\ DANS LA DESCENDANCE DE TAUREAUX CULARDS \\ SUIVANT LE TYPE GÉNÉTIQUE DES MÈRES : \\ RÉSULTATS PARTIELS
}

F. Ménissier et B. Bibé. - Station de Génétique quantitative et appliquée, C. N.R.Z. I. N. R. A., 78-Jouy-en-Josas (France).

L'utilisation de taureaux culards présente un intérêt évident pour améliorer la production de viande fournie par des femelles de races rustiques dans le cadre de schémas de croisement simple ou double.

Nous avons étudié la fréquence d'apparition de ce caractère dans la descendance de taureaux charolais (CC) et blonds d'Aquitaine (BB) de ce type accouplés avec des femelles de races rustiques ou croisées dans le cadre de 2 essais. Le premier essai comportait des femelles Aubrac (AA) et croisées de première génération avec des mâles des races à viande ci-dessus exprimant $\left(A C_{C}\right.$ et $\left.A B_{C}\right)$ ou non $\left(A C_{N}\right.$ et $\left.A B_{N}\right)$ le caractère culard. Le deuxième essai était réalisé sur des génisses de race gasconne (GG) et croisées de première génération avec des mâles charolais ou blonds d'Aquitaine (GC et $\mathrm{GB}$ ) de phénotype inconnu; nous disposions en outre, dans ce deuxième essai, de femelles charolaises et blondes d'Aquitaine de phénotype normal (CC) et $(\mathrm{BB})$. Le caractère culard était apprécié subjectivement sur l'ensemble des veaux $(65$ et 73 respectivement pour chaque essai) issus de ces accouplements. Ces derniers étaient répartis en 2 catégories : normaux d'une part, culards et semi-culards d'autre part.

Tous les veaux croisés de I $^{\text {re }}$ génération peuvent être considérés comme normaux.

Les variations de fréquence du caractère dans la descendance des femelles croisées sont peu influencées par la race paternelle (CC ou $B B)$ par le type de croisement $\left(A_{N}\right.$ et $A B_{N}$, GC et GB) ; toutefois, les femelles croisées issues d'un père culard fournissent un pourcentage de veaux culards très supérieur à celui des femelles issues d'un taureau normal :

$$
\begin{aligned}
& \mathrm{AC}_{\mathrm{C}}=75 \text { p. Ioo et } \mathrm{ACN}=\text { o p. roo } \\
& \mathrm{AB}_{\mathrm{C}}=40 \text { p. Ioo et } \mathrm{ABN}=8 \text { p. Ioo }
\end{aligned}
$$

